\title{
A novel method to obtain proximal plethysmographic information from distal measurements using the impedance plethysmogram
}

\author{
J. Gomez-Clapers ${ }^{1,2}$, R. Casanella ${ }^{1}$, and R. Pallas-Areny ${ }^{1}$ \\ 1. Instrumentation, Sensors and Interfaces Group, Electronic Engineering Department, UPC-BarcelonaTech, Castelldefels, Spain \\ 2.E-mail any correspondence to: joan.gomez-clapers@upc.edu
}

\begin{abstract}
We present a novel method to detect proximal volume changes based on the impedance plethysmogram (IPG) measured from limb to limb with two electrode pairs symmetrically placed at distal areas of the upper or the lower limbs. Since the measurement is sensitive to changes along the whole current path, this method allows us to detect changes in arteries that are more proximal to the torso than the measurement sites. Our results show that the Pulse Arrival Time (PAT) measured from the R peak of the ECG to the hand-to-hand IPG is close to the PAT to the elbow whereas the PAT measured from the R peak of the ECG to the foot-to-foot IPG is close to the PAT to the knee. This opens new avenues for noninvasive cardiovascular measurements based only on electrodes in contact with hands or feet.
\end{abstract}

Keywords: IPG, PTT, limbs, cardiovascular monitoring

\section{Introduction}

The increasing prevalence of cardiovascular diseases over the last decades is forcing healthcare systems to shift its focus to early detection and preventive therapies, in order to reduce the overall spending on treatments and to decrease their impact on patients' lives [1]. However, traditional technologies for screening and diagnosing are not well suited to this new scenario since these often rely on the availability of limited resources such as expensive devices and highly trained staff. Consequently, a heavy effort is currently being devoted to the conception of novel reliable and cost-effective technologies suitable for performing fast screenings and regular self-administrated health status checks at home or in secondary health facilities, which better suit the present needs of healthcare systems.

Arterial stiffness has been identified as a key factor for assessing cardiovascular disease risk [2,3] since it can be obtained noninvasively in ambulatory environments from the Pulse Wave Velocity (PWV), i.e. the propagation speed of the pressure pulse generated by the cardiac ejection of blood from the heart to the arterial tree on each heartbeat. The timing delay in the arrival of the pressure pulse between a proximal and a distal site, the so-called Pulse Transit Time (PTT), is generally accepted as the most simple, robust, and reproducible method to obtain the PWV [4], which can be calculated from the PTT according to

$$
\mathrm{PWV}=\frac{D}{\mathrm{PTT}}
$$

where $D$ is the distance between the two recording sites measured on the body surface. Photoplethysmography (PPG) [5], impedance plethysmography (IPG) [6], mechanical transducers [7], and arterial tonography [8] are the most common technologies for PTT measurements [9] because they are simple, non-invasive, and cost-effective.

Nevertheless, PTT measurements using these common technologies require time to expose and clean a proximal and a distal site, and to place the sensors and wires, which is a nuisance for fast screenings or regular selfadministrated health status checks. This preparation time can be reduced by placing the sensors in areas that are habitually uncovered or easily accessible, such as the distal parts of the limbs [6]. However, short distances $D$ between sensors yield low-accuracy PTT measurements hence the placement of the proximal sensor onto an area proximal to the torso is often required, and that area is less accessible than the hands or feet. Further, PTT measurements in central arteries such as the aorta, which show the highest predictive value for cardiovascular risk assessment [10], include large portions of other arteries when performed with common pressure-pulse recording devices placed at the limbs and this reduces the measurement performance [11] hence measurements in areas proximal to the torso are more advisable.

In addition to common measurements along a single artery, the IPG can also be measured from hands to feet, the so-called whole-body impedance plethysmogram [12]. Even though the specific contribution of the different body segments in the current path is unknown, whole-body IPG waveforms precede the IPG obtained on the foot, which indicates that the IPG upstroke forcefully reflects plethysmographic changes in proximal sites with respect to the recording sites hence the PTT can be derived from these two signals. However, the method proposed in [12] requires the exposure of four recording sites and the placement of six electrodes, which is still unpractical for fast screenings or regular self-administrated health status checks.

Alternatively, the IPG can be measured from hand to hand [13] and from foot to foot [14], which only requires the exposure of two measurement sites. Since the current injected in limb-to-limb measurements travels through proximal and distal arteries, it is reasonable to assume that certain features of the recorded waveform will reflect volume changes in arteries more proximal to the torso than 
the recording site, analogously to whole-body impedance measurements. This fact was first observed in the signals obtained from a four-electrode system intended for measuring the Pulse Arrival Time (PAT) [15], which is defined as the time delay between the electrical activation of the heart and the arrival of the pressure pulse to a specific body site.

In this paper, we compare the timing of the hand-tohand IPG $\left(\mathrm{IPG}_{\mathrm{hh}}\right)$ and the foot-to-foot IPG (IPG $\left.\mathrm{ff}_{\mathrm{ff}}\right)$ with several local IPG waveforms simultaneously obtained with two close electrodes placed on the target area, in order to investigate the suitability of limb-to-limb IPG measurements to obtain proximal plethysmographic information by using four electrodes placed only on distal sites.

\section{Materials and methods}

\section{IPG acquisition system}

The current injection system to obtain the IPG was a Howland current source connected to a $20 \mathrm{kHz}$ sine-wave oscillator, with $0.5 \mathrm{~mA}$ peak current, as shown in Fig.1.

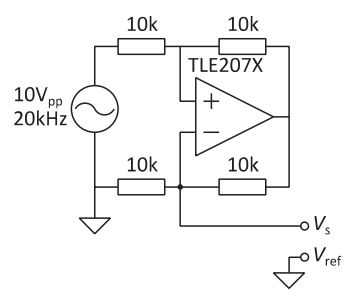

Fig.1: IPG current injection system.

The voltage drop was measured with an instrumentation amplifier, high-pass-coupled through a first-order high-pass differential filter [16] with $f_{\mathrm{c}}=90 \mathrm{~Hz}$ to reject input offset voltage and power line interference, and gain set to 10 for limb-to-limb impedance measurements and to 50 for local impedance measurements. The output voltage was demodulated with a coherent demodulator implemented by a $+1 /-1$ gain amplifier, whose output was fed back to the reference terminal of the instrumentation amplifier through an integrator (Fig. 2). This yields an equivalent first-order high-pass response with $f_{\mathrm{c}}=0.15 \mathrm{~Hz}$, which is the recommended high-pass cutoff frequency for pressure-pulse measurements [17]. High-frequency components at the demodulator output were rejected by a second-order lowpass Sallen-Key filter with gain set to 220 and $f_{\mathrm{c}}=20 \mathrm{~Hz}$, which is the recommended low-pass cutoff for pressurepulse signals [18].

\section{Measurement setup}

The hand-to-hand IPG and the foot-to-foot IPG were evaluated in two separate experiments. In each set of measurements, each of the two current injection electrodes was placed on the distal part of one limb of a subject, thus

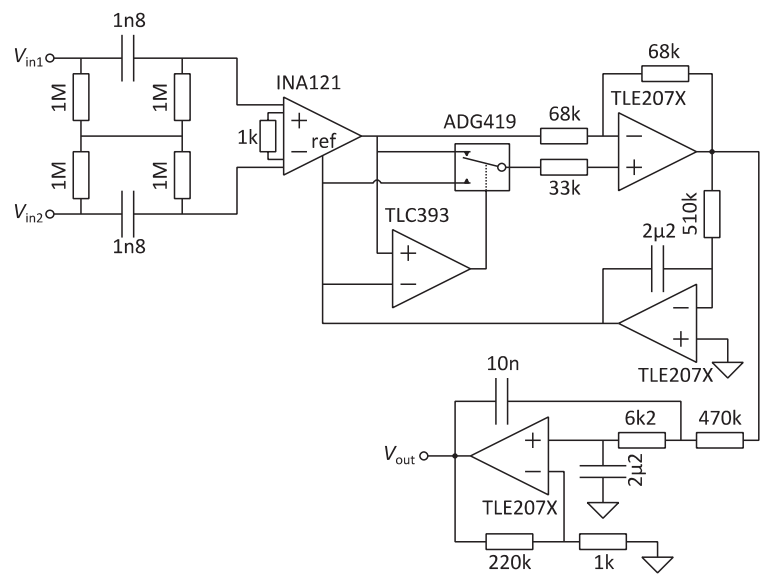

Fig.2: IPG voltage measurement system.

forcing the current to flow from one limb to another, and the local and limb-to-limb IPG were measured by using the same injected current. In order to evaluate the arrival times of the pressure pulse at different locations, the two electrodes to obtain the local IPG were sequentially placed onto different measurement sites. During the experiments, an additional ECG was simultaneously obtained from an IPG-compatible system [15] in order to provide a timing reference for the recorded waveforms.

For the hand-to-hand IPG test, the current was injected from the left index finger to the right index finger and the $\mathrm{IPG}_{\mathrm{hh}}$ was measured between the left middle finger and the right middle finger. The ECG was obtained from the same electrodes connected to the middle fingers, as described in [15]. The local IPG was sequentially recorded on the shoulder (IPG1), elbow (IPG2), wrist (IPG3), and the index finger (IPG4) with an inter-electrode distance of about $10 \mathrm{~cm}$ between the recording pair. Fig. 3 shows the setup for the hand-to-hand IPG test.

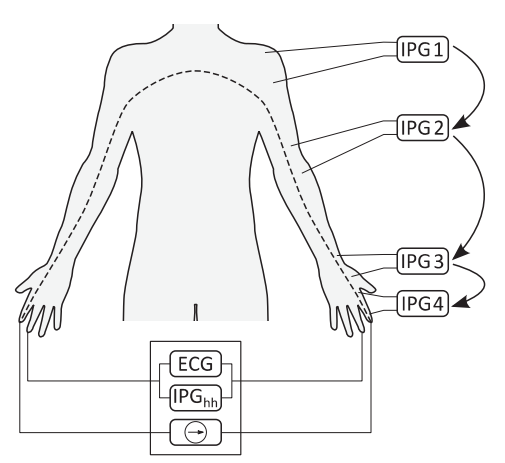

Fig.3: Setup for the hand-to-hand IPG test.

For the foot-to-foot IPG test, the current was injected from the left forefoot to the right forefoot and $\mathrm{IPG}_{\mathrm{ff}}$ was measured between the left heel and the right heel. The ECG was obtained from two electrodes connected to the hands. The local IPG was sequentially recorded from the femoral artery at the mid-inguinal point (IPG5), knee (IPG5), and the ankle (IPG7) with an interelectrode distance of about $10 \mathrm{~cm}$ between the recording pair. Fig. 4 shows the setup for the foot-to-foot IPG test. 


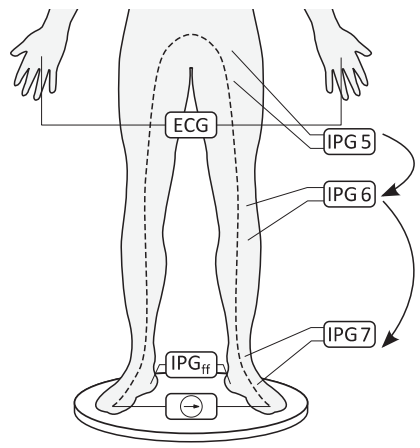

Fig.4: Setup for the foot-to-foot IPG test.

\section{Signal processing}

The $\mathrm{R}$ peak of the ECG, identified by the Pan-Tompkins algorithm [19], was used as a time reference $(t=0)$ for the arrival time of the measured waveforms hence the timings reported below correspond to the PAT. The arrival of the pressure-pulse was determined from the timing of the foot of the wave identified by the intersecting tangents method due to its superior performance as compared to other methods [20].

Noise and artifacts in the recorded waveforms were reduced by using a 5-heartbeat sample average aligned from the ECG R peak. To avoid the effect of hemodynamic changes during the sequential recordings of local IPG waveforms that could thwart the interpretation of the results, the PAT to the limb-to-limb waveform, which was continuously recorded during the experiments, was used as an indicator of hemodynamic changes and only the samples in which this value was constant were considered.

In order to easily compare the IPG signals, the amplitude of the waveforms was normalized.

\section{Results}

Fig. 5 shows the traces of the IPG $_{h h}$ and the local IPG obtained at the shoulder, elbow, wrist, and the index finger.

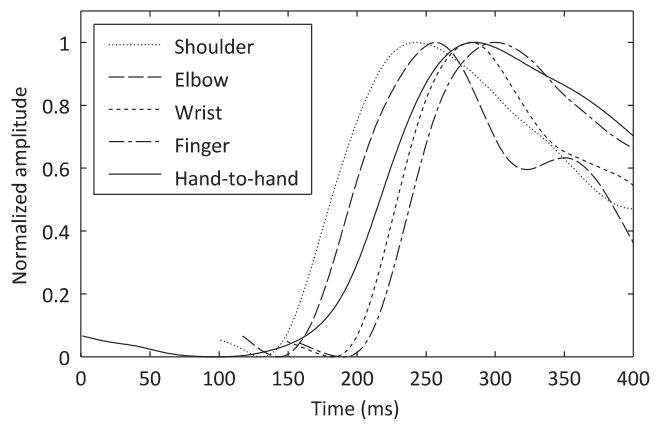

Fig.5: Hand-to-hand IPG and local IPG at the shoulder, elbow, wrist, and the index finger.

Compared to the local IPG waveforms, which have similar foot and upstroke shapes and only differ in the arrival time, the $\mathrm{IPG}_{\mathrm{hh}}$ waveform is smoother, especially in the area of the foot. The minimum of the signal precedes any of the other local IPGs but the leading edge comes after that of the elbow IPG and the peak coincides with that of the wrist
IPG. The PAT to the $\mathrm{IPG}_{\mathrm{hh}}$ using the tangent intersection method is $178 \mathrm{~ms}$, whereas the PAT to the shoulder, elbow, wrist, and the finger using the same method are $148 \mathrm{~ms}$, $164 \mathrm{~ms}, 201 \mathrm{~ms}$, and $210 \mathrm{~ms}$, respectively.

Fig. 6 shows the traces of the IPG $_{\mathrm{ff}}$ and the local IPG obtained at the femoral artery, knee, and the ankle.

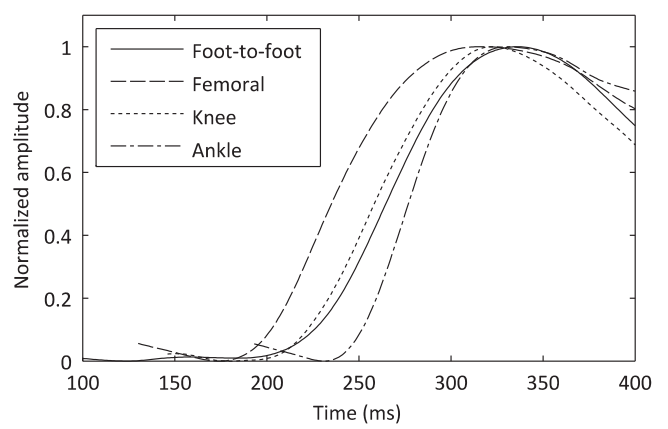

Fig.6: Foot-to-foot IPG and local IPG at the femoral artery, knee, and the ankle.

Similar to the IPG $\mathrm{Ih}_{\mathrm{h}}$, the foot of the $\mathrm{IPG}_{\mathrm{ff}}$ is smoother than that of local IPG waveforms and the upstroke arrives later, but less than in the IPG $\mathrm{Gh}_{\mathrm{hh}}$. The minimum of the $\mathrm{IPG}_{\mathrm{ff}}$ signal and its maximal slope are located close to those of the knee IPG, and the peak of the $\mathrm{IPG}_{\mathrm{ff}}$ comes later than that of the ankle IPG. The PAT to the $\mathrm{IPG}_{\mathrm{ff}}$ is $236 \mathrm{~ms}$, whereas the PAT to the femoral artery, knee, and the ankle are $198 \mathrm{~ms}$, $220 \mathrm{~ms}$, and $250 \mathrm{~ms}$, respectively.

\section{Discussion}

The measured PAT to the IPG $\mathrm{Hh}_{\mathrm{h}}$ and the $\mathrm{IPG}_{\mathrm{ff}}$ were significantly lower than the PAT to the local IPG obtained at the different measurement sites in the arm and leg. The values reported with respect to other local pulse-pressure waveforms suggest that the PAT to the $\mathrm{IPG}_{\mathrm{hh}}$ could be used as a surrogate of the PAT to the elbow whereas the PAT to the $\mathrm{IPG}_{\mathrm{ff}}$ could be used as a surrogate for the PAT to the knee. Combined with a local pressure-pulse waveform, it is possible to measure a PTT of $32 \mathrm{~ms}$ at the hands and a PTT of $14 \mathrm{~ms}$ at the feet, which are longer PTT values than those in other IPG measurements [6] in spite of being measured in a smaller area.

Further, the longer delay between the minimum of the signal and the maximal slope of the upstroke in limb-tolimb IPG waveforms as compared to the local IPG waveforms suggests that common algorithms to detect the foot of the limb-to-limb IPG signal that rely on features of the upstroke, such as the tangent intersection, could be unsuitable. This could explain the discrepancy between PAT values here measured with the IPG $\mathrm{Ih}_{\mathrm{hh}}$ and PAT values measured with a finger PPG as reported in [15], where the pressure-pulse arrival is determined by the mid-raise of the upstroke. Since the finger PPG arrives later than the foot of the $\mathrm{IPG}_{\mathrm{hh}}$ determined by the intersecting tangents method, it is reasonable to assume that it will yield longer timings in limb-to-limb waveforms due to the slower slope of the upstroke. 


\section{Conclusions}

We have studied the suitability of limb-to-limb measurements performed with two distal electrode pairs for assessing proximal plethysmographic information with respect to the recording sites. Our results show that the foot of the wave detected by the intersecting tangents method is close to the pressure-pulse arrival at the elbow for the IPG $\mathrm{Hh}_{\mathrm{hh}}$ and to the pressure-pulse arrival at the knee for the $\mathrm{IPG}_{\mathrm{ff}}$, which allows us to obtain PTT values of $32 \mathrm{~ms}$ at the hands and $14 \mathrm{~ms}$ at the feet when combined with a distal pressurepulse waveform. These PTT values are longer than those obtained by current methods. This is remarkable as the limb-to-limb IPG only requires the exposure of two easily accessible recording sites at the distal parts of the limbs. Further, our results suggest that limb-to-limb signals should yield better aortic PTT measurements than other methods that use signals obtained at the limbs because these include a larger contribution of distal arteries.

A limitation of this study is that the performance of limb-to-limb IPG waveforms under hemodynamic variations has not been explored. Future work should focus on determining the accuracy of the pressure pulse timing under diverse maneuvers intended to induce hemodynamic changes and in a wider group of test subjects to test the intra-subject variability. Another area of future work could be the development of methods to detect the foot of limbto-limb IPG signals as their leading edge is different from that of local IPG signals.

This notwithstanding, limb-to-limb IPG systems can open new opportunities to design reliable and cost-effective methods to enable fast PTT measurements in clinic and ambulatory environments.

\section{Acknowledgements}

This project was funded by the Secretaría de Estado de Investigación, Desarrollo e Innovación del Gobierno de España and the European Regional Development Fund, under the project TEC2009-13022 and the grants BES2010-032893 and EEBB-I-13-06744.

\section{References}

1. World Health Organization (WHO). Global Atlas of Cardiovascular Disease Prevention and Control. Geneve: 2011.

2. Vlachopoulos C, Aznaouridis K, Terentes-Printzios D, Ioakeimidis N, and Stefanadis C. Prediction of Cardiovascular Events and All-cause Mortality With Brachial-ankle Elasticity Index: A Systematic Review and Meta-analysis. J Hypertens. 2012; 60(2): 556-62.

http://dx.doi.org/10.1161/HYPERTENSIONAHA.112.194779

3. The Reference Values for Arterial Stiffness' Collaboration. Determinants of Pulse Wave Velocity in Healthy People and in the Presence of Cardiovascular Risk Factors: Establishing Normal and Reference Values. Eur Heart J. 2010; 31(19): 2338-50. http://dx.doi.org/10.1093/eurheartj/ehq165
4. Laurent S, Cockcroft J, Van Bortel L, Boutouyrie P, Giannattasio C, et al. Expert Consensus Document on Arterial Stiffness: Methodological Issues and Clinical Applications. Eur Heart J. 2006; 27(21): 2588-605. http://dx.doi.org/10.1093/eurheartj/eh1254

5. Allen J. Photoplethysmography and Its Application in Clinical Physiological Measurement. Physiol Meas. 2007; 28(3): R1R39. http://dx.doi.org/10.1088/0967-3334/28/3/R01

6. Risacher F, Jossinet J, McAdams ET, McLaughlin J, Mann Y, et al. Impedance Plethysmography for the Evaluation of Pulse Wave Velocity in Limbs. Med Biol Eng Comput. 1993; 31(3): 318-22. http://dx.doi.org/10.1007/BF02458053

7. Asmar R, Benetos A, Topouchian J, Laurent P, Pannier B, et al. Assessment of Arterial Distensibility by Automatic Pulse Wave Velocity Measurement: Validation and Clinical Application Studies. J Hypertens. 1995; 26(3): 485-90. http://dx.doi.org/10.1161/01.HYP.26.3.485

8. Salvi P, Lio G, Labat C, Ricci E, Pannier B, et al. Validation of a New Non-Invasive Portable Tonometer for Determining Arterial Pressure Wave and Pulse Wave Velocity: The PulsePen Device. J Hypertens. 2004; 22(12): 2285-93. http://dx.doi.org/10.1097/00004872-200412000-00010

9. Buxi D, Redouté JM, and Yuce MR. A Survey on Signals and Systems in Ambulatory Blood Pressure Monitoring Using Pulse Transit Time. Physiol Meas. 2015; 36(3): R1-R26. http://dx.doi.org/10.1088/0967-3334/36/3/R1

10. Van Bortel LM, Laurent S, Boutouyrie P, Chowienczyk P, Cruickshank JK, et al. Expert Consensus Document on the Measurement of Aortic Stiffness in Daily Practice Using Carotid-femoral Pulse Wave Velocity. J Hypertens. 2012; 30(3): 445-8. http://dx.doi.org/10.1097/HJH.0b013e32834fa8b0

11. Wilkinson IB, McEniery CM, Schillaci G, Boutouyrie P, Segers P, et al. ARTERY Society Guidelines for Validation of Non-invasive Haemodynamic Measurement Devices: Part 1, Arterial Pulse Wave Velocity. Artery Res. 2010; 4(2): 34-40. http://dx.doi.org/10.1016/j.artres.2010.03.001

12. Kööbi T, Kähönen M, Iivainen $T$, and Turjanmaa V. Simultaneous Non-invasive Assessment of Arterial Stiffness and Haemodynamics - A Validation Study. Clin Physiol Funct Imaging. 2003; 23(1): 31-6. http://dx.doi.org/10.1046/j.1475-097X.2003.00465.x

13. Casas O and Pallas-Areny R. Signal to Noise Ratio in Bioelectrical Impedance Measurements Using Synchronous Sampling. Ann IEEE Int Conf Eng Med Biol Soc. 1994: 8901. http://dx.doi.org/10.1109/iembs.1994.415198

14. Gonzalez-Landaeta R, Casas O, and Pallas-Areny R. Heart Rate Detection From Plantar Bioimpedance Measurements. IEEE Trans Biomed Eng. 2008; 55(3): 1163-7. http://dx.doi.org/10.1109/TBME.2007.906516

15. Gomez-Clapers J, Casanella R, and Pallas-Areny R. Pulse Arrival Time Estimation From the Impedance Plethysmogram Obtained With a Handheld Device. Ann IEEE Int Conf Eng Med Biol Soc. 2011: 516-9. http://dx.doi.org/10.1109/iembs.2011.6090093

16. Spinelli EM, Pallas-Areny R, and Mayosky MA. AC-coupled Front-end for Biopotential Measurements. IEEE Trans Biomed Eng. 2003; 50(3): 391-5. http://dx.doi.org/10.1109/TBME.2003.808826 
17. Allen J and Murray A. Effects of Filtering on Multi-site Photoplethysmography Pulse Waveform Characteristics. Computers in Cardiology 2004: 485-88. http://dx.doi.org/10.1109/cic.2004.1442980

18. Peura R. Blood Pressure and Sound. In: Webster JG editor: Medical Instrumentation Application and Design. New York: John Wiley \& Sons; 2009: 293-337.
19. Pan J and Tompkins WJ. A Real-time QRS Detection Algorithm. IEEE Trans Biomed Eng. 1985; 32(3): 230-6. http://dx.doi.org/10.1109/TBME.1985.325532

20. Millasseau SC, Stewart AD, Patel SJ, Redwood SR, and Chowienczyk PJ. Evaluation of Carotid-femoral Pulse Wave Velocity: Influence of Timing Algorithm and Heart Rate. J Hypertens. 2005; 45(2): 222-6. http://dx.doi.org/10.1161/01.HYP.0000154229.97341.d2 